\title{
Cervical cancer screening in low-resource settings: a smartphone image application as an alternative to colposcopy
}

This article was published in the following Dove Press journal:

International Journal of Women's Health

22 June 2017

Number of times this article has been viewed

\author{
Caroline Gallay ${ }^{1, *}$ \\ Anne Girardet ${ }^{1, *}$ \\ Manuela Viviano ${ }^{2}$ \\ Rosa Catarino ${ }^{2}$ \\ Anne-Caroline Benski ${ }^{2,3}$ \\ Phuong Lien Tran ${ }^{2,4}$ \\ Christophe Ecabert ${ }^{5}$ \\ Jean-Philippe Thiran ${ }^{5}$ \\ Pierre Vassilakos ${ }^{6}$ \\ Patrick Petignat ${ }^{2}$ \\ 'Faculty of Medicine, University \\ of Geneva, Geneva, Switzerland; \\ ${ }^{2}$ Department of Gynecology and \\ Obstetrics, Geneva University \\ Hospitals, Geneva, Switzerland; \\ ${ }^{3}$ Department of Gynecology and \\ Obstetrics, Saint Damien Healthcare \\ Centre, Madagascar; ${ }^{4}$ Department of \\ Gynecology and Obstetrics, Reunion \\ University Hospitals, Reunion Island, \\ France; ${ }^{5}$ Signal Processing Laboratory \\ (LTS5), Ecole Polytechnique Fédérale \\ de Lausanne (EPFL), Lausanne, \\ Switzerland; ${ }^{6} \mathrm{Geneva}$ Foundation \\ for Medical Education and Research, \\ Geneva, Switzerland
}

*These authors have contributed equally to the work
Correspondence: Phuong Lien Tran

Department of Gynecology and

Obstetrics, Geneva University

Hospitals, Boulevard de la Cluse 30,

1205 Geneva, Switzerland

Tel +4I 795535452

Fax +4I 223724188

Email phuong_lien_tran@yahoo.com
Background: Visual inspection after application of acetic acid (VIA) and Lugol's iodine (VILI) is a cervical cancer (CC) screening approach that has recently been adopted in low- and middle-income countries (LMIC). Innovative technologies allow the acquisition of consecutive cervical images of VIA and VILI using a smartphone application. The aim of this study was to evaluate the quality of smartphone images in order to assess the feasibility and usability of a mobile application for CC screening in LMIC.

Methods: Between May and November 2015, women aged 30-65 years were recruited in a CC screening campaign in Madagascar. Human papillomavirus-positive women were invited to undergo VIA/VILI assessment. Pictures of their cervix were taken using a Samsung Galaxy S5 with an application called "Exam", which was designed to obtain high-quality images and to classify them in the following sequence: native, VIA, VILI and posttreatment. Experts in colposcopy were asked to evaluate if the quality of the pictures was sufficient to establish the diagnosis and to assess sharpness, focus and zoom.

Results: The application use was simple and intuitive, and 208 pictures were automatically classified and recorded in the patient's file. The quality was judged as adequate for diagnosis in $93.3 \%$ of cases. The interobserver agreement was $\kappa=0.45(0.23-0.58)$, corresponding to a moderate agreement on the common scale of kappa values.

Conclusion: This smartphone application allows the acquisition of good quality images for VIA/VILI diagnosis. The classification of images in a patient database makes them accessible to on- and off-site experts, and allows continuous clinical education. Smartphone applications may offer an alternative to colposcopy for CC screening in LMIC.

Keywords: visual approach, cervical cancer screening, human papillomavirus, HPV, smartphone, mobile health

\section{Introduction}

Approximately $85 \%$ of cervical cancer (CC) cases worldwide occur in low- and middle-income countries (LMIC), ${ }^{1}$ mainly due to the lack of efficient primary and secondary prevention programs. ${ }^{2,3}$ The CC incidence in sub-Saharan Africa is approximately 31.5 per 100,000 women, accompanied by a mortality rate of approximately 17.9 per 100,000 women, which makes CC the most common cancer and the most common cause of cancer-related death among African women. ${ }^{4}$ The recommended screening approach in LMIC for CC is visual inspection of the cervix after the application of acetic acid (VIA), which has been approved by the World Health Organization, and is often followed by the application of Lugol's iodine (VILI). ${ }^{5}$ Visual inspection methods are simple, inexpensive and require minimal infrastructure, allowing patients 
to be screened and treated within the same-day session. ${ }^{6}$ Nevertheless, the diagnosis made with this method remains subjective as its quality is affected by intrapersonal limitations, such as the experience and the training degree of the examiner, as well as external factors, such as the lighting conditions and the woman's cervical anatomy.

Monitoring the quality of a health program ensures the systems' optimization and maximizes the benefit of the target population. Quality control activities for CC screening include the implementation of accuracy verification systems directed toward optimization of the screening tests' performance. In many LMIC, human resources may not be readily available, particularly if health facilities are located in remote, hard-to-reach areas. ${ }^{7}$

In this context, the development of an image-based system that could assist clinicians in the diagnosis of cervical precancer may represent an important step toward the improvement of bare eye-based visual inspection screening techniques. As colposcopes are expensive, electricity dependent and need high maintenance, several alternatives, such as smartphonebased technologies, have been developed. A project outlining the feasibility of VIA/VILI to replace colposcopy has been previously published. ${ }^{8}$ The smartphone-based approach opens up new possibilities, thanks to its accessibility, userfriendly interfaces and high-definition cameras to picture the cervix. ${ }^{9}$ Moreover, sharing images with long-distance experts in real time allows health care providers to obtain advice and to improve their work's quality. The use of smartphone applications as a tool to minimize the diagnosis subjectivity has been experienced in other medical fields, such as that of dermatology for the detection of skin melanoma. ${ }^{10-12} \mathrm{By}$ offering a quality control system, the use of images taken with a smartphone may support the CC screening process. ${ }^{13}$

The aim of this study was to evaluate the quality of smartphone images taken during a CC screening campaign in LMIC in order to assess the feasibility and usability of a mobile health (m-health) application for CC screening in LMIC.

\section{Methods}

\section{Study design and enrollment}

This study took place between May and November 2015 at the Saint Damien Health Care Center, Ambanja, Madagascar. This town, with a population of more than 33,000 inhabitants, is located in the northern region of Madagascar. Since 2010, the Saint Damien Health Care Center has periodically run routine human papillomavirus (HPV)-based CC screening campaigns recruiting 1,000 women each year.
Criteria for inclusion in the study were women aged 30-65 years and able to comply with the study protocol. Exclusion criteria were pregnancy beyond 20 weeks and former conisation or hysterectomy. All eligible women were asked to sign a written informed consent form before taking part in the study. Ethical approval was obtained from the Malagasy National Commission for the Ethics of Science and Technology and from the Ethical Cantonal Board of Geneva, Switzerland (CER: 14-071. Registered as a Clinical Trial - NCT02693379). A total of 784 women were recruited in the study. Self-collected vaginal samples were promptly analyzed for HPV with the GeneXpert machine (GeneXpert ${ }^{\circledR} I V$ Cepheid, 2015; Sunnyvale, CA, USA), and the results were given to the participants on the same day.

The sample size was determined based on the hypothesis that the quality of smartphone images would be considered good in $85 \%$ of the cases, with a two-tailed $\alpha=0.05$ and $1-\beta=0.80$. Taking into consideration a population size of approximately 33,000 inhabitants and that three to four images would be taken for each women, we set the total sample size to 200 images. Thus, 56 patients fulfilling inclusion criteria were invited to participate in the smartphone study. They benefited from VIA/VILI assessment and further evaluation with the smartphone application, allowing the capture of 208 photos. The flowchart of the study is represented in Figure 1.

\section{"Exam", an m-Health application}

Pictures of the cervix were taken with a Samsung Galaxy S5 (Samsung, Seoul, South Korea) using an application named "Exam". It is an android application specifically designed to obtain high-quality images by the Signal Processing Laboratory of the Swiss Federal Institute of Technology in Lausanne, Switzerland. This application's functioning is based on the fact that the changes of the cervical surface induced by the application of acetic acid and Lugol's iodine can be captured by inexpensive smartphone cameras simply by recording multiple sequential images of the cervix immediately after the application of each solution. The image capture, featured extraction and classification methods are implemented to run directly on an Android smartphone, using the Software Development Kit made available by Google to develop Android applications.

\section{Data collection and image capture}

Consecutive images were captured for the respectively included patients without any selection or exclusion criteria. Patients were positioned in lithotomic position, the speculum was inserted and vaginal discharge was removed with a dry 


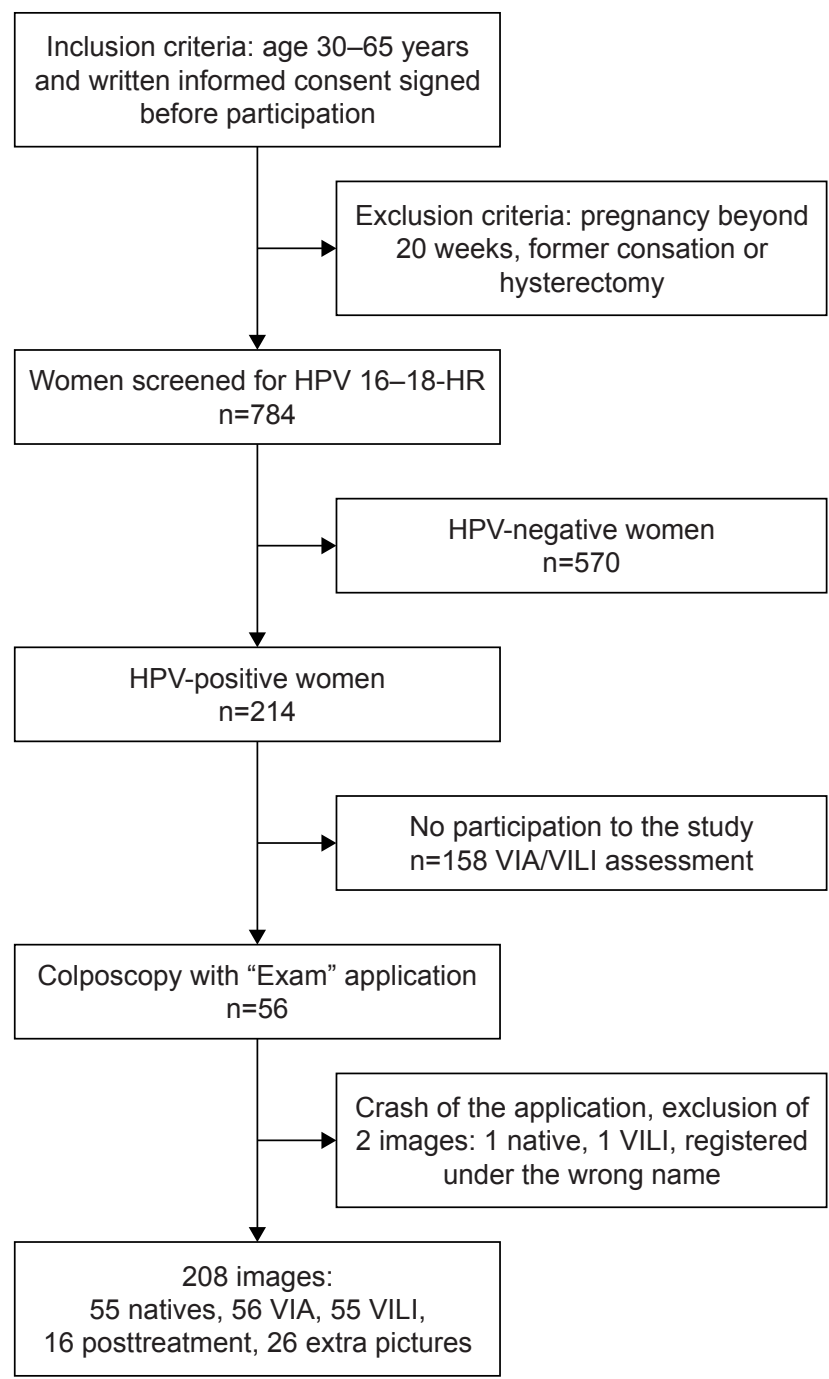

Figure I Study flowchart.

Abbreviations: HPV, human papillomavirus; HR, high risk; VIA, visual inspection after application of acetic acid; VILI, visual inspection after application of Lugol's iodine.

or saline solution-soaked cotton swab. The smartphone was held up at a $15 \mathrm{~cm}$ distance from the vulva using a tripod, whose position could be modified on three axes-height, depth and rotation-in order to ensure its maximum stability and to allow the acquisition of the best possible images in terms of quality. The tripod could be inclined in order to be as close as possible to the cervix (at a minimum distance of $\sim 1.5 \mathrm{~cm}$ from the speculum). Figure 2 illustrates the main components of the device, including the gynecological table, mobile phone and tripod.

Prior to each examination, the tripod was placed and adjusted in front of the patient. The patient's examination was then registered in the "Exam" application.

Once the patient record was created, the cervical images were acquired in a precise sequence: native, VIA, VILI and posttreatment whenever this was performed. The flash

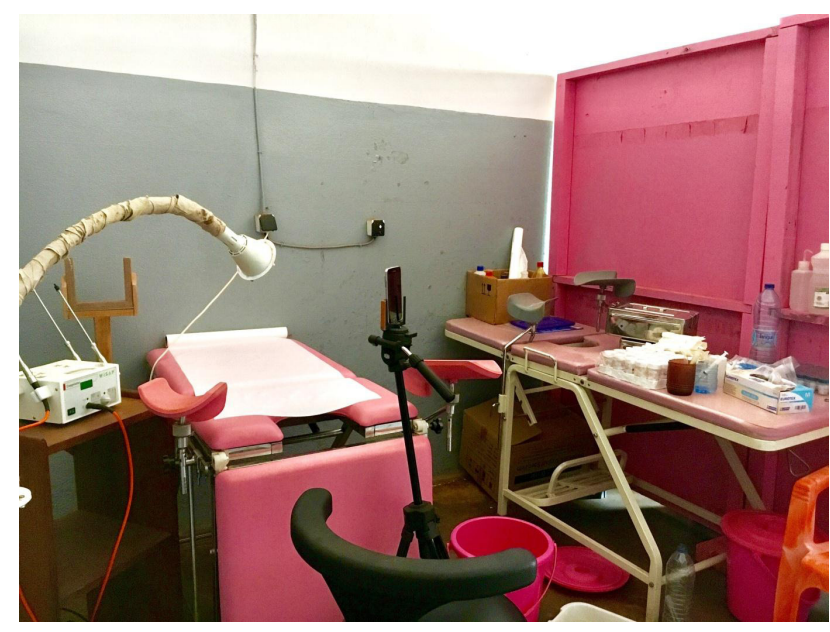

Figure 2 Device including the gynecological table, smartphone and tripod.

was set as on, and the zoom button on the left of the screen allowed us to zoom in between 1.39 and 1.69 times during the procedure. A target in the center of the screen allowed us to focus on the cervix with the intent of standardizing the exam. Figure 3 illustrates the different steps as they appear on the smartphone's screen.

The first, native picture of the cervix was taken. Then the application left us some time to apply acid acetic and capture the VIA picture. In the same method, after Lugol's iodine was applied, the VILI picture was taken. Finally, Exam proposed to take a picture once treatment was performed, otherwise it allowed the user to close the program. Furthermore, it offered the possibility at each step to either take an extra photo ("picture more") or retake the same photo ("replace a picture"). If necessary, the tripod could be repositioned and adjusted between each step. The pictures were automatically saved and classified in the patient's file by date and type (native, VIA, VILI, posttreatment). Written comments could be added to each patient's visual file. Finally, the biopsy site was marked on the VIA picture with a cross mark. The VIA picture could also be viewed without the marked biopsy site.

All the pictures collected by the smartphone application were sent to a central database. For security and privacy, data transfer to the central database was accomplished using an encrypted key. Authentication was required to access the patients' file, and only the caregivers who received accreditation with a personal identifier and password could log into the smartphone application or the respective database. Access to patient data was only possible after scanning a bar code unique for each patient or by entering the patient's identification number and the date of the VIA/VILI assessment. This ensured patients' information to be protected. 


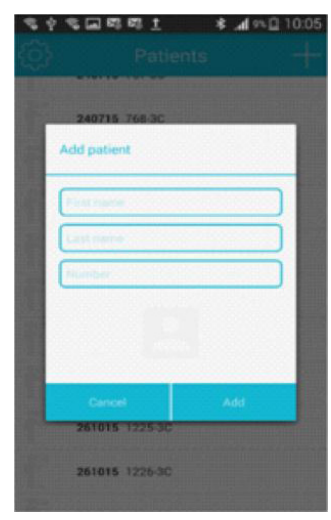

Step 1

Register the patient in the application

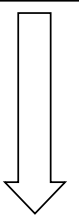

Step 2

Take a picture of the native cervix

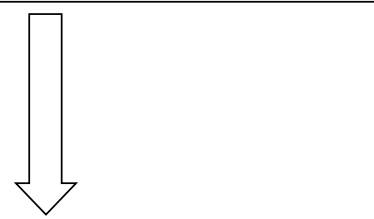

Step 3

Apply acetic acid. Once VIA is done, click on the button and continue to take the VIA picture
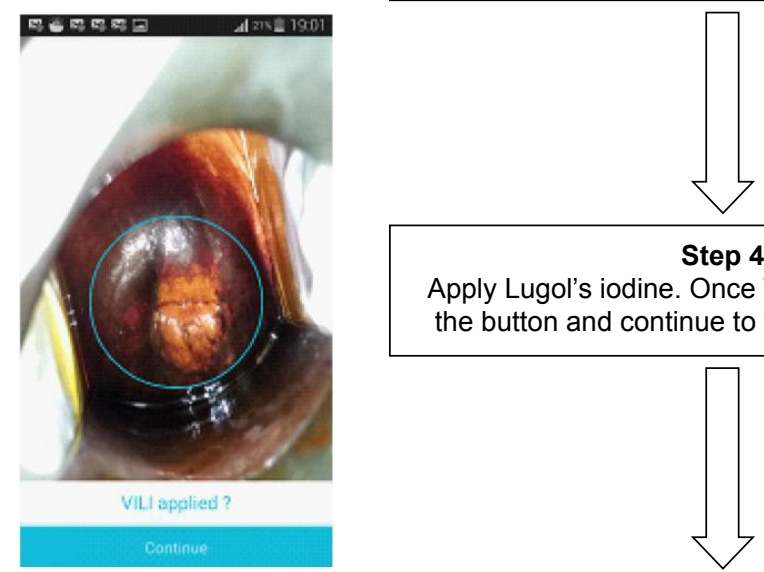

\section{Step 4}

Apply Lugol's iodine. Once VILI is done, click on the button and continue to take the VILI picture

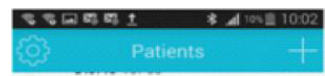

$$
\begin{aligned}
& 240715760-3 \mathrm{C} \\
& 240715 \text { min: } 3 \mathrm{C} \\
& \text { 251015 } 1192 \cdot 3 \mathrm{C} \\
& 261015+105-36 \\
& 2610151207 \cdot 3 \mathrm{C} \\
& 2610151212-3 \mathrm{C} \\
& \text { 261015 1222:x } \\
& \text { 261015 1225.3C } \\
& 2610151226-36
\end{aligned}
$$

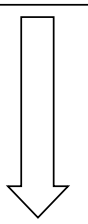

Step 5

If no treatment is necessary, click No and the application will close down. If treatment is recommended, treat and click Continue to take the posttreatment photo
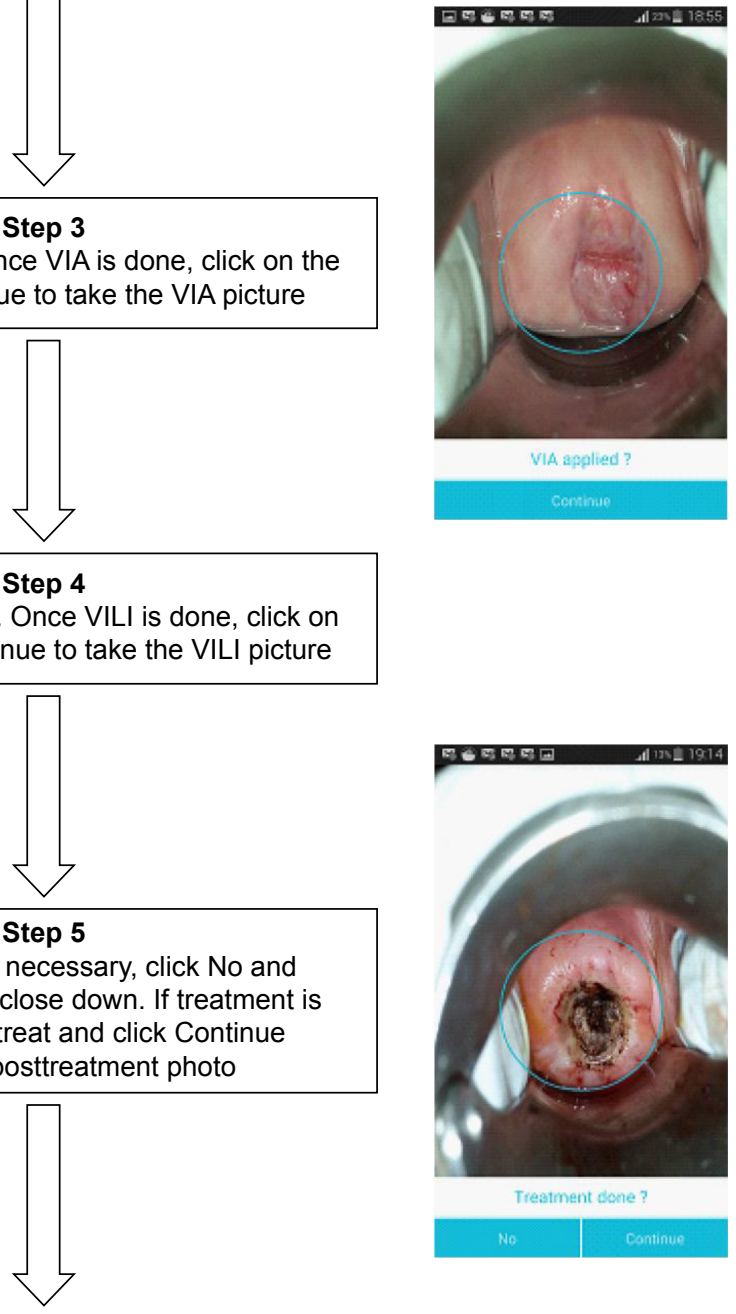

Each patient's image file is saved and can be retrieved by using the date of primary screening, the patient's number of inclusion in the screening campaign and the patient's first of last name

Figure 3 Images' acquisition flowchart.

Abbreviations: VIA, visual inspection after application of acetic acid; VILI, visual inspection after application of Lugol's iodine.

\section{Pictures' quality evaluation and statistical analysis}

The pictures' quality was assessed independently by three expert gynecologists in colposcopy (more than 10 years' experience and more than 200 colposcopies per year for each of them). For each photo, they had to determine whether the quality of the three images allowed the diagnosis according to the following criteria:

- Diagnostic utility, depending on how helpful the image was for the treatment decision making 
- Sharpness, determining the amount of detail one image could convey, with absence of blurring

- Focus, defined as the sharpness on the cervix

- Zoom, characterized as the presence of the cervix in the middle of the picture, occupying at least one-third of the picture's surface

For each criterion, the answer was binary: satisfying or not (score 1 or 0 , respectively).

A global score (sum of the four criteria) was then calculated for each picture based on the fulfillment of these four criteria. The indicator for judging the results of the co-assessment was the interrater agreement for the images' quality.

What defines a good picture is the content of the picture, evaluated by diagnostic utility and sharpness, and technical characteristics assessed by focus and zoom. Thus, we judged each photo to be of good quality whenever at least two criteria were fulfilled. We then calculated the consensus when at least two physicians obtained the same score. Thus, kappa $(\kappa)$ values were calculated for each physician in order to estimate the agreement between the physician's evaluation and that of the consensus.

Statistical analyses were run with a statistical software package (Stata statistical software: release 14; StataCorp. College Station, TX, USA).

\section{Results}

\section{Photo quality evaluation and interrater agreement}

A total of 208 photos were evaluated by three physicians, resulting in 624 evaluations. All criteria were fulfilled in the majority of pictures. The aspect fulfilled in most cases was the focus (89.1\%), followed by the diagnostic utility (83.7\%), the sharpness $(77.7 \%)$ and the zoom $(73.7 \%)$. These results are reported in Table 1.

According to the consensus, 194/208 images (93.27\%) were judged as being of good quality, sufficient for diagnosis, while only $14 / 208(6.73 \%)$ were judged to be of poor quality (Table 2).

Table I Pictures fulfilling the four quality criteria of a total of 624 evaluations

\begin{tabular}{|c|c|c|c|c|}
\hline & $\begin{array}{l}\text { Sharpness } \\
\text { n (\%) }\end{array}$ & $\begin{array}{l}\text { Focus } \\
\text { n (\%) }\end{array}$ & $\begin{array}{l}\text { Zoom } \\
\text { n (\%) }\end{array}$ & $\begin{array}{l}\text { Diagnostic } \\
\text { utility } \\
\text { n (\%) }\end{array}$ \\
\hline Rater I & I43 (68.8) & I84 (88.5) & I49 (7I.6) & $150(72.1)$ \\
\hline Rater 2 & I5I (72.6) & $166(79.8)$ & $116(55.8)$ & $166(79.8)$ \\
\hline Rater 3 & I91 (91.8) & $206(99.0)$ & $195(93.8)$ & $206(99.0)$ \\
\hline Total & 485 (77.7) & $556(89.1)$ & $460(73.7)$ & $522(83.7)$ \\
\hline
\end{tabular}

Table 2 Evaluation of photo quality (two or more of the following criteria fulfilled: focus diagnostic utility, sharpness and zoom)

\begin{tabular}{lll}
\hline & $\begin{array}{l}\text { Good quality photos } \\
\text { n (\%) }\end{array}$ & $\begin{array}{l}\text { Poor quality photos } \\
\text { n (\%) }\end{array}$ \\
\hline Rater I & $177(85.1)$ & $31(14.9)$ \\
Rater 2 & $176(84.6)$ & $32(15.4)$ \\
Rater 3 & $206(99.0)$ & $2(1.0)$ \\
Consensus & $194(93.3)$ & $14(6.7)$ \\
\hline
\end{tabular}

The agreement between each physician and the consensus concerning the picture quality as calculated with the $\kappa$ value, ranged from a minimum of 0.23 to a maximum of 0.58 . The overall agreement obtained with light's correction for $\mathrm{K}$ was 0.45 , corresponding to a "moderate agreement" on the common scale of $\kappa$ values (Table 3 ).

\section{Feasibility of the application}

Among the images taken by the application, there were 55 natives, 56 VIA, 55 VILI, 16 posttreatment and 26 extra pictures. Due to a sudden crash of Exam during the clinical visit, two pictures (1 native, 1 VILI) were registered under the wrong name and were therefore excluded from the analysis. All pictures were saved in the phone's memory card and classified by date and type (native, VIA, VILI, posttreatment and extra) in the patient's image file, thus creating a database for both on- and off-site patient information retrieval.

For each examination, since the examiner could choose to replace a picture or to take an extra picture if he/she was disappointed with the first picture, a mean of 4.8 photographs was necessary in order to obtain satisfying images. Once the pictures were saved on the computer, they were classified in the same way as they were on the smartphone: by the patient's identification number, by the date of the VIA/VILI assessment and by the picture type.

\section{Discussion}

The major asset of this m-Health application for $\mathrm{CC}$ screening is the quality of the images for VIA/VILI diagnosis. The physicians judged most cervical images to be of good quality and thus capable of guiding them throughout the decision-making

Table 3 Agreement and kappa values between each rater and the consensus

\begin{tabular}{llllll}
\hline & $\begin{array}{l}\text { Obtained } \\
\text { agreement } \\
(\%)\end{array}$ & $\begin{array}{l}\text { Expected } \\
\text { agreement } \\
(\%)\end{array}$ & $\kappa$ & $\begin{array}{l}\text { Standard } \\
\text { error }\end{array}$ & P-value \\
\hline Rater I & 91.83 & 80.37 & 0.58 & 0.06 & $<0.001$ \\
Rater 2 & 90.38 & 79.96 & 0.52 & 0.06 & $<0.001$ \\
Rater 3 & 94.23 & 92.44 & 0.23 & 0.05 & $<0.001$ \\
\hline
\end{tabular}


process that requires them to promptly opt for a clinical management pathway. The capture of high-quality digital images of the cervix allows health care providers to save the patient's image and to retrieve it once the examination is completed. This practical retrieval of information can be used to empower frontline workers to become more effective through virtual and dynamic training. Moreover, this smartphone application allows the physician to eventually seek another expert's opinion either on- or off-site, thus fulfilling the purpose of helping make the diagnostic and, more importantly, clinical management decision. By comparing the diagnoses of health care providers and their supervisor(s), this co-assessment approach ensures quality control of the screening and treatment decision-making process.

Furthermore, this m-Health application is able to easily classify the photos, which facilitates their retrieval while guaranteeing the correct match between the image file and the patient's identity. The application generates a picture database containing the images of all patients who underwent VIA/VILI assessment, while guaranteeing the absence of data loss. The images are automatically classified in the sequence of native, VIA, VILI and posttreatment, which corresponds to the order in which these colposcopy steps are taken in the everyday practice, thereby respecting the sequential logic of the original examination. Due to the smartphone efficiency and ease of handling, the database is readily accessible, regardless of the presence of the computer. The application saves all images on the smartphone's memory card, which makes them easily transferable onto a computer using a USB cable allowing, therefore, to quickly create a backup database.

This smartphone application currently has some limitations that need to be addressed. Throughout the visit, the application does not allow the user to go back and forth between the different images if the sequence is not yet complete, thus possibly reducing the added benefit to the clinical decision. The proposed solution is to add a "gallery" icon on the screen that would allow the examiner to compare side by side the photos acquired during the ongoing procedure. This aspect is currently being evaluated by the Federal Institute of Technology located in Lausanne, Switzerland. The incorporation of a green light is also under discussion for future updates.

Although some manipulations are acceptable, the tripod requires more handling than expected in order to capture the cervix from the best possible angle. The limited freedom of movement can be explained by the localization of the tripod between the patient's legs, which is intrinsically a narrow space that allows limited amplitude of movements. This aspect could be improved through increasing experience, which would allow health care providers to become progressively more familiar with the practice of smartphone-based visual inspection techniques.

As a consequence of the prohibitive cost of colposcopes (US\$10,000-\$20,000), VIA is performed with a simple headlamp in most resource-limited settings. ${ }^{14}$ Nevertheless, the performance of naked eye VIA as a CC screening tool is far from optimal. Such limitation translates into high screen positivity rates that are likely due to inadequate training and that inevitably lead to high overtreatment rates. By providing an efficient and practical quality control system, the smartphone-based VIA approach circumvents the main issues that limit the naked eye VIA's efficacy. A previous study showed that the overall agreement between the diagnoses' quality obtained from live colposcopic visualization and static digital imaging of the cervix was $43.0 \%$ corresponding to a moderate agreement. ${ }^{15}$ Hence, further improvement of the application is imperative in order to optimize its performance as a $\mathrm{CC}$ screening tool. The ultimate aim is to replace the colposcopebased pelvic examination with a smartphone-based one, thus extending CC screening to those resource-constrained areas who have no access to a standard colposcope and who are likely to suffer the most from the consequence of reduced access to health care.

\section{Conclusion}

The use of a smartphone application that allows the acquisition of images during the pelvic examination is feasible in LMIC. The Exam application is able to capture high-quality images and is an efficient method for storing the patients' data. As the overall pictures' quality was judged as good, this smartphone-based approach can potentially be integrated in the context of CC screening and, in the long run, could offer an alternative to colposcopy for CC screening in LMIC. Further studies are needed to evaluate the quality of the images, including VIA/VILI assessment in the treatment decision making.

\section{Disclosure}

The authors report no conflicts of interest in this work.

\section{References}

1. Bray F, Ren JS, Masuyer E, Ferlay J. Global estimates of cancer prevalence for 27 sites in the adult population in 2008. Int J Cancer. 2013; 132(5):1133-1145.

2. Quinn M, Babb P, Jones J, Allen E. Effect of screening on incidence of and mortality from cancer of cervix in England: evaluation based on routinely collected statistics. BMJ. 1999;318(7188):904-908. 
3. UpToDate [website on Internet]. Screening for cervical cancer [updated Apr 06, 2016]. Available from: http://www.uptodate.com/contents/ screening-for-cervical-cancer?source $=$ search_result $\&$ search $=$ screenin $\mathrm{g}+$ for+cervical+cancer \&selectedTitle $=1 \% 7 \mathrm{E} 150$. Accessed January 16, 2017.

4. Ferlay J, Soerjomataram I, Dikshit R, et al. Cancer incidence and mortality worldwide: sources, methods and major patterns in GLOBOCAN 2012. Int J Cancer. 2015;136(5):E359-E386.

5. UpToDate [website on Internet]. Cervical cancer screening tests: Visual inspection methods [updated Jan 19, 2016]. Available from: http:// www.uptodate.com/contents/cervical-cancer-screening-tests-visualinspection-methods?source=search_result\&search $=$ cervical+cancer + screening+tests+visual+inspection+meth+ods\&selectedTitle $=1 \sim 150$. Accessed January 16, 2017.

6. World Health Organization. Prevention of cervical cancer through screening using visual inspection with acetic acid (VIA) and treatment with cryotherapy. Geneva: World Health Organization; 2012. Available from: http:/www.who.int/reproductivehealth/publications/ cancers/9789241503860/en/. Accessed January 16, 2017.

7. World Health Organization/Pan American Health Organization. Monitoring national cervical cancer prevention and control programmes: quality control and quality assurance for visual inspection with acetic acid (VIA)-based programmes. Geneva: World Health Organization; 2013. Available from: http://www.who.int/reproductivehealth/publications/cancers/9789241505260/en/. Accessed January 16, 2017.

8. Quinley KE, Gormley RH, Ratcliffe SJ, et al. Use of mobile telemedicine for cervical cancer screening. J Telemed Telecare. 2011;17(4): 203-209.
9. Smith A. Nearly half of American adults are smartphone owners. On: Pew Research Center: Internet, Science \& Tech [webpage on Internet]; 2012. Available from: http://www.pewinternet.org/2012/03/01/nearlyhalf-of-american-adults-are-smartphone-owners/. Accessed January 16, 2017.

10. Maier T, Kulichova D, Schotten K, et al. Accuracy of a smartphone application using fractal image analysis of pigmented moles compared to clinical diagnosis and histological result. J Eur Acad Dermatol Venereol. 2015;29(4):663-667.

11. March J, Hand M, Grossman D. Practical application of new technologies for melanoma diagnosis: part I. Noninvasive approaches. $\mathrm{J} \mathrm{Am}$ Acad Dermatol. 2015;72(6):929-941.

12. Arnhold M, Quade M, Kirch W. Mobile applications for diabetics: a systematic review and expert-based usability evaluation considering the special requirements of diabetes patients age 50 years or older. $J$ Med Internet Res. 2014;16(4):e104.

13. Ricard-Gauthier D, Wisniak A, Catarino R, et al. Use of Smartphones as Adjuvant Tools for Cervical Cancer Screening in Low-Resource Settings. J Low Genit Tract Dis. 2015;19(4):295-300.

14. Lam CT, Krieger MS, Gallagher JE, Asma B, Muasher LC, Schmitt JW, et al. Design of a Novel Low Cost Point of Care Tampon (POCkeT) Colposcope for Use in Resource Limited Settings. PLoS One. 2015;10(9):e0135869.

15. Liu AH, Gold MA, Schiffman M, et al. Comparison of Colposcopic Impression Based on Live Colposcopy and Evaluation of Static Digital Images. J Low Genit Tract Dis. 2016;20(2):154-161.
International Journal of Women's Health

\section{Publish your work in this journal}

The International Journal of Women's Health is an international, peerreviewed open-access journal publishing original research, reports, editorials, reviews and commentaries on all aspects of women's healthcare including gynecology, obstetrics, and breast cancer. The manuscript management system is completely online and includes

\section{Dovepress}

a very quick and fair peer-review system, which is all easy to use. Visit http://www.dovepress.com/testimonials.php to read real quotes from published authors. 